\title{
Inflammatory Myofibroblastic Tumor of the Urinary Bladder in a Young Patient With Gross Hematuria: A Case Report
}

\author{
Han Kyu Chae', Wook Nam ${ }^{1}$, Sung Jin Kim¹, Byeong-Joo Noh², Jong Yeon Park ${ }^{1}$ \\ 'Department of Urology, Gangneung Asan Medical Center, University of Ulsan College of Medicine, Gangneung, Korea \\ ${ }^{2}$ Department of Pathology, Gangneung Asan Medical Center, University of Ulsan College of Medicine, Gangneung, Korea
}

Inflammatory myofibroblastic tumor (IMT) of the urinary bladder is an uncommon disease in young patients that presents with gross hematuria. However, IMT should be cautiously diagnosed because of its similarities with malignant spindle cell sarcoma. In this case, the tumor was successfully removed by transurethral resection of the bladder tumor and a final diagnosis of IMT was established conclusively.

Key Words: Urinary bladder, Inflammatory myofibroblastic tumor
Received October 8, 2021

Revised December 1, 2021

Accepted December 5, 2021

Corresponding author:

Jong Yeon Park

Email: jypark@gnah.co.kr

https://orcid.org/0000-0002-8657-1474
Inflammatory myofibroblastic tumor (IMT) of the urinary bladder was first reported by Roth in 1980 as an unusual pseudosarcomatous lesion. ${ }^{1}$ Our 33-year-old male patient was diagnosed with IMT of the urinary bladder.

\section{CASE REPORT}

A 33-year-old man consulted the urology clinic of our institute with a 1-month history of painless gross hematuria. The patient had no previous disease history but reported that his grandfather had died of bladder cancer. An abdominopelvic computed tomography (APCT) scan revealed a round, $3.6-\mathrm{cm}$ nodular mass around the right dome of the urinary bladder without significant perivesical fat infiltration (Fig. 1A, B). Cystoscopy demonstrated the mass on the bladder dome (Fig. 1C). Transurethral resection of the bladder tumor
(TURBT) was performed, and histopathologic examination for the resected deep bladder muscle revealed myofibroblastic spindle cells intermingled with inflammatory cells, delineating haphazardly arranged fascicles within areas of variable (Fig. 2). Immunohistochemical (IHC) stains demonstrated diffuse expression of anaplastic lymphoma kinase 1 (ALK1) (Fig. 3A), a focal expression of desmin (Fig. 3B) and smooth muscle actin (SMA) (Fig. 3C). Meanwhile, c-Kit and S-100 were negative. Based on the pathological and IHC findings, the diagnosis is consistent with IMT. The patient is undergoing outpatient follow-up until 6 months without signs of tumor recurrence.

\section{DISCUSSION}

According to the 2020 World Health Organization definition, IMT is a rare tumor composed of myo- 


\section{KJUO}
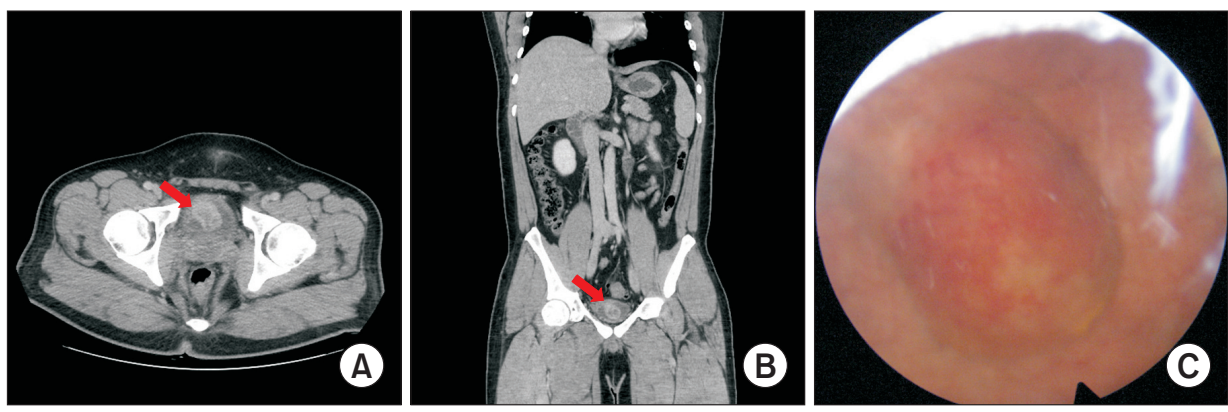

Fig. 1. (A, B) Enhanced abdominopelvic computed tomography showed an enhanced 3.6-cm-sized bladder mass (red arrows). (C) Cystoscopy revealed a single solid erythematous tumor at the dome of the bladder.
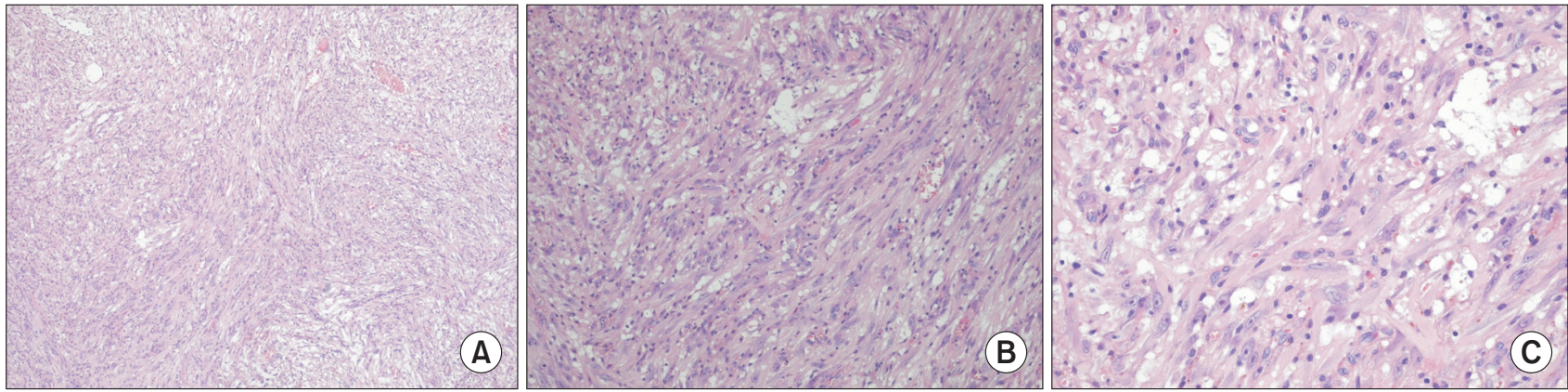

Fig. 2. Light microscopy of inflammatory myofibroblastic tumor shows eosinophilic cytoplasm with indistinct nuclear borders and nuclear pleomorphism. Hematoxylin and eosin stain: (A) $\times 40$ magnification, $(B) \times 100$ magnification, $(C) \times 200$ magnification.
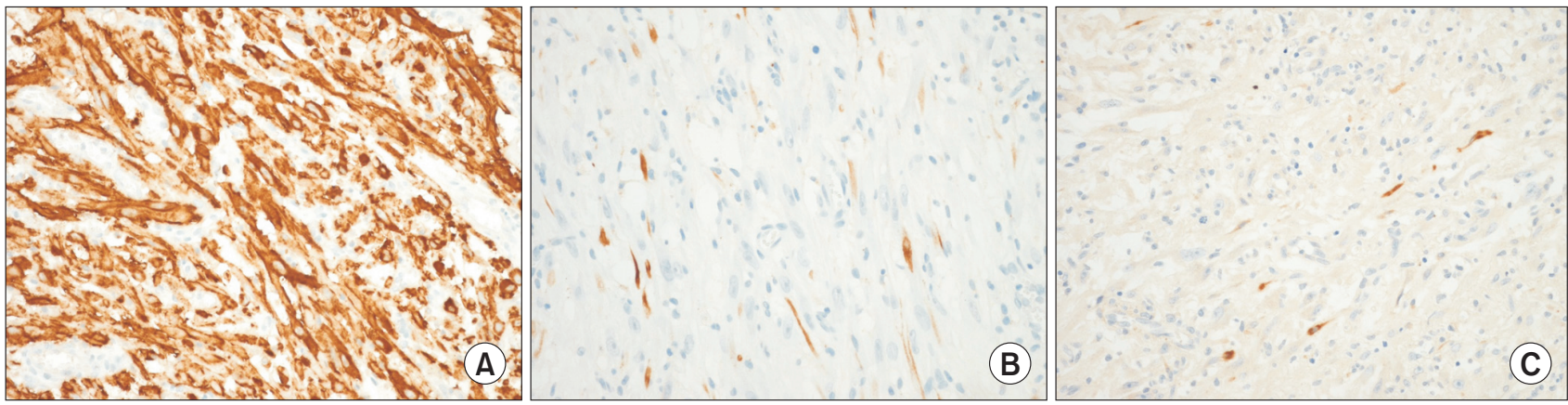

Fig. 3. Immunohistochemical staining of inflammatory myofibroblastic tumor. (A) ALK1 cytoplasmic marker $(\times 200$ magnification), (B) Desmin cytoplasmic marker $(\times 200$ magnification), (C) Smooth muscle actin cytoplasmic marker $(\times 200$ magnification).

fibroblasts and fibroblast spindle cells, characterized by inflammatory plasma cell, eosinophil, and lymphocyte infiltration. ${ }^{2}$ The presence of ALK1, SMA, vimentin, and cytokeratin is the most wellknown immunohistochemical feature of IMT spindle cells. ${ }^{3,4}$

Although IMT is reportedly common in young individuals, bladder IMT is common in adults in their third and fourth decades of life. ${ }^{5}$ Its most common clinical feature is gross hematuria, followed by lower urinary tract symptoms. ${ }^{6}$ Although hemodynamic instability due to hematuria is uncommon, severe hematuria can require transfusion and emergency TURBT. ${ }^{7}$

TURBT is the preferred treatment for the complete surgical removal of IMT. Although radical cystectomy and systemic chemotherapy have been used due to disease extent and malignant transformation, ${ }^{8,9}$ 
bladder preservation should be considered for its benign nature. Advanced diagnostics using ALK1/ p80 immunohistochemistry and fluorescence in situ hybridization analysis has increased diagnostic accuracy, resulting in successful bladder-preserving surgery even in locally advanced cases. ${ }^{10}$

Although the true incidence is unknown, IMT reportedly has excellent prognosis. No literature was found that compared and analyzed the differences in treatment and prognosis according to age. However, Li et al. ${ }^{11}$ reported 11 children diagnosed with urinary tract IMT, 8 patients (72.7\%) were treated with local tumor resection alone, and 3 patients (27.3\%) had segmental resection. In all cases, there was no recurrence during the follow-up period. According to a study analyzing 14 adults aged over 20 years, 7 cases achieved complete remission with TURBT alone, and partial cystectomy was performed in the remaining cases, and radical cystectomy was also performed in one case. ${ }^{7}$ Based on this, it can be inferred that the prognosis is relatively good in children, but it is necessary to analyze with larger number of patients. Teoh et al. $^{6}$ assessed 182 IMT patients with urinary bladder among 41 studies and reported a local recurrence rate of $4 \%$ with a median $30.0 \pm 28.2$ months of follow-up. Our patient has been followed up without recurrence until 6 months after the initial TURBT. Regular follow-up is necessary because of recurrence rates of up to $25 \% .^{5}$ Wang et al. ${ }^{12}$ recommended cystoscopy and APCT every 3-6 months within the first year of follow-up and repeat cystoscopy every 6 months for the first 2 years.

IMT studies reported to date and our patient's clinical features suggest that, in cases of hematuria at a relatively young age without other risk factors, cystoscopy should always be performed and the condition should not be misdiagnosed as chronic cystitis. If a solid bladder mass is identified on cystoscopy at a site other than the trigone, bladder preservation requires consideration. IMT should be excluded through immunohistochemical analysis, without prioritizing the possibility of malignancy, such as leiomyosarcoma.

Herein, we reported the case of a young patient with IMT. Although easy to treat, IMT should always be considered since its pathological findings can easily be mistaken for sarcoma. Regular followup is required due to possible recurrence and metastasis.

\section{NOTES}

- Conflicts of interest: No potential conflict of interest relevant to this article was reported.

- Funding: This study received no specific grant from any funding agency in the public, commercial, or not-for-profit sectors.

- ORCID

Han Kyu Chae: https://orcid.org/0000-0002-3082-5842

Wook Nam: https://orcid.org/0000-0002-1424-9339

Sung Jin Kim: https://orcid.org/0000-0002-5609-0485

Byeong-Joo Noh: https://orcid.org/0000-0003-3288-4157

Jong Yeon Park: https://orcid.org/0000-0002-8657-1474

\section{REFERENCES}

1. Roth JA. Reactive pseudosarcomatous response in urinary bladder. Urology 1980;16:635-72.

2. Kallen ME, Hornick JL. The 2020 WHO classification: what's new in soft tissue tumor pathology? Am J Surg Pathol 2021;45:e1-23.

3. Reinhart S, Trachsel Y, Fritz C, Wagner U, BodeLesniewska B, John H, et al. Inflammatory myofibroblastic tumor of the bladder with FN1-ALK gene fusion: different response to ALK inhibition. Urology 2020;146:32-5.

4. Harik LR, Merino C, Coindre JM, Amin MB, Pedeutour F, Weiss SW. Pseudosarcomatous myofibroblastic proliferations of the bladder: a clinicopathologic study of 42 cases. Am J Surg Pathol 
2006;30:787-94.

5. Montgomery EA, Shuster DD, Burkart AL, Esteban JM, Sgrignoli A, Elwood L, et al. Inflammatory myofibroblastic tumors of the urinary tract: a clinicopathologic study of 46 cases, including a malignant example inflammatory fibrosarcoma and a subset associated with high-grade urothelial carcinoma. Am J Surg Pathol 2006;30:1502-12.

6. Teoh JY, Chan NH, Cheung HY, Hou SS, Ng CF. Inflammatory myofibroblastic tumors of the urinary bladder: a systematic review. Urology 2014;84:5038.

7. Song D, Jiao W, Gao Z, Liu N, Zhang S, Zong Y, et al. Inflammatory myofibroblastic tumor of urinary bladder with severe hematuria: a case report and literature review. Medicine (Baltimore) 2019;98: e13987.

8. Kim HW, Choi YH, Kang SM, Ku JY, Ahn JH, Kim JM, et al. Malignant inflammatory myofibroblastic tumor of the bladder with rapid progression. Korean J Urol 2012;53:657-61.

9. Libby EK, Ellis LT, Weinstein S, Hammer RD, Murray KS. Metastatic inflammatory myofibroblastic tumor of the bladder. Urol Case Rep 2019;23:10-2.

10. Kato M, Masui S, Kanda H, Arima K, Shiraishi T, Sugimura Y. Successful preservation of the bladder in a case of inflammatory myofibroblastic tumor with the diagnostic efficacy of ALK/p80 immunohistochemistry and FISH analysis: case report and review of the literature. Urol Case Rep 2017;11:1921.

11. Li YP, Han WW, Yang Y, He LJ, Zhang WP. Inflammatory myofibroblastic tumor of the urinary bladder and ureter in children: experience of a tertiary referral center. Urology 2020;145:229-35.

12. Wang W, Zhang M, Lu JR, Li QY. Inflammatory myofibroblastic tumor of urinary bladder. Chin Med J (Engl) 2018;131:2259-61. 